\title{
Fatores estressores e as estratégias de enfrentamento utilizadas por enfermeiros hospitalares: revisão'
}

Priscilla Cavalcante Lima²

Kerolaynne Cardoso Vieira Sabino²

Márcia Teles de Oliveira Gouveia³

Fernanda Valéria Silva Dantas Avelino 3

Márcia Astrês Fernandes 3

Como citar: Lima P, Sabino KC, Gouveia M, Avelino FV, Fernandes M. Fatores estressores e as estratégias de enfrentamento utilizadas por enfermeiros hospitalares: revisão Investig Enferm. Imagen Desarr. 2015;17(2): 51-65. http://dx.doi.org/10.11144/Javeriana.ie17-2.feea

1. Artigo derivado de pesquisa. Recebido: 8 de setembro de 2014. Aceito: 5 de dezembro de 2014. Disponivel on-line: 2 de maio de 2015.

2. Graduadas em Enfermagem pela Universidade Federal do Piauí (UFPI), Teresina-PiauíBrasil. Correio-e: priclima90@gmail.com; kerolcvieira@hotmail.com

3. Enfermeira. Doutora em Enfermagem. Professora da Universidade Federal do Piauí (UFPI), Teresina-Piaui-Brasil. Correio-e: marcia06@gmail.com; fvavelino@gmail.com; m.astres@ bol.com.br 


\section{Resumo}

Objetivos: analisar a produção científica referente aos principais fatores estressores e as estratégias de enfrentamento utilizadas por enfermeiros hospitalares. Metodologia: trata-se de uma revisão integrativa; realizada em julho de 2013 nos bancos de dados LILACS, Embase e SciELO, resultando na seleção de 16 publicações. Discussão: o estresse é natural da condição humana e pode ser desencadeado por diversos fatores que estão direta ou indiretamente ligados ao ambiente hospitalar e para que o estresse não se torne patológico existem as estratégias de coping. Conclusões: com o estudo percebeu-se que não existe uma única estratégia de coping efetiva e que as estratégias devem ser associadas entre si para o enfrentamento do estresse.

Palavras-chave: coping; estresse ocupacional; enfermeiros

\section{Los factores de estrés y las estrategias de afrontamiento utilizadas por las enfermeras que laboran en hospitales: revisión}

\section{Resumen}

Objetivos: analizar la literatura científica de los principales factores de estrés y estrategias de afrontamiento utilizadas por las enfermeras en el hospital. Metodologia: revisión integrativa que se realizó en julio de 2013 en las bases de datos LILACS, Embase y SciELO. Se encontraron 15 publicaciones que cumplian con los criterios de inclusión. Discusión: el estrés es natural y la condición humana puede ser provocada por varios factores que están directa o indirectamente vinculados con el hospital, por lo cual se requieren estrategias de afrontamiento. Conclusiones: en el estudio se observó que no hay una estrategia única y para que sean eficaces deberían estar asociados entre sí para hacer frente al estrés.

Palabras clave: afrontamiento; estrés laboral; enfermeras

\section{Stressors and coping strategies used by nurses working in the hospital: review}

\section{Abstract}

Objectives: To analyze the scientific literature of the major stressors and coping strategies used by nurses in the hospital. Methodology: Integrative review held in July 2013 in LILACS, Embase, and SciELO data bases. 15 publications that met the inclusion criteria were found. Discussion: stress is natural and the human condition can be caused by several factors that are directly or indirectly linked to the hospital, therefore coping strategies are required. Conclusions: the study found that there is no a single strategy to be effective and those strategies should be associated among them in order to be effective in coping with stress.

Keywords: coping; work stress; nurses 


\section{Considerações iniciais}

O mundo contemporâneo e as novas exigências do mercado exigem profissionais cada vez mais especializados e capacitados. Para atender a essas exigências, os profissionais precisam desdobrar-se desenvolvendo várias atividades diárias, mas o dinamismo e a inconstância do mercado acabam gerando tensões, medo e estresse, afetando a qualidade de vida, as tarefas diárias e a interação com as pessoas no ambiente de trabalho.

Os profissionais da saúde, em especial os enfermeiros, são constantemente confrontados com exigências nem sempre possíveis de serem cumpridas e que provocam frequentemente o estresse, que é entendido como um estímulo advindo do ambiente interno ou externo e que excede a capacidade de adaptação do indivíduo. Fatores como a carga excessiva de trabalho, jornada dupla, reduzido número de profissionais e baixos salários, acarretam risco à saúde do trabalhador e à organização, pois geram baixo desempenho, alta rotatividade e absenteísmo (1-2).

$\mathrm{Na}$ tentativa de contornar as tensões, os enfermeiros utilizam estratégias conhecidas como coping, definidas como um processo dinâmico de esforços direcionados para a resolução das dificuldades. Coping envolve uma gama de estratégias comportamentais e cognitivas que podem ser usadas para alterar, evitar e reavaliar circunstâncias estressantes ou aliviar seus efeitos. Essas estratégias variam de acordo com o objetivo desejado e incluem intervenções voltadas para o indivíduo, para as relações indivíduo-organização e para o trabalho em equipe (3).

Durante a graduação, os acadêmicos passam por diversos campos de estágio, onde é possivel identificar os fatores desencadeantes do estresse presentes no ambiente hospitalar. Contudo, não ficam claras as estratégias utilizadas por enfermeiros para enfrentar, contornar ou evitar os fatores estressantes que os afetam. O estudo teve como objetivo analisar a produção científica referente aos principais fatores estressores e as estratégias de enfrentamento utilizadas por enfermeiros hospitalares.

\section{Metodologia}

A pesquisa trata de uma revisão integrativa, que tem como objetivo a análise de pesquisas, possibilitando a sintese e explicitando as lacunas do conhecimento existente em uma área particular de estudo (4).

A condução da revisão integrativa foi realizada em seis etapas: identificação do tema e seleção da hipótese ou questão de pesquisa para a elaboração da revisão integrativa; amostragem ou busca na literatura; definição das informações a serem extraídas dos estudos selecionados; avaliação dos estudos incluídos na revisão integrativa; interpretação dos resultados e apresentação da revisão/sintese do conhecimento (4).

Considerando a temática do estudo, elegeram-se então as seguintes questões norteadoras para guiar a pesquisa: quais os principais fatores estressores para os enfermeiros no ambiente hospitalar? Quais as principais estratégias de coping utilizadas por enfermeiros para enfrentar o estresse? Baseada nas perguntas norteadoras foi realizada a pesquisa nos bancos de dados para a seleção dos artigos. 
A busca foi desenvolvida em julho de 2013, nos bancos de dados indexados: Literatura Latino Americana em Ciências da Saúde (LILACS), Embase e Scientific Eletronic Library Online (SciELO), utilizando os descritores em ciências da saúde (DeCs): coping, estresse ocupacional e enfermeiros. Foram critérios de inclusão dos artigos: terem sido publicados entre janeiro de 2008 a julho de 2013, no idioma português e que abordassem a temática do estudo. Foram excluídos os artigos de revisão, as teses, os editoriais, as dissertações, artigos que não estavam disponiveis na întegra e que não abordavam no seu título e resumo a temática do estudo.

A busca por artigos na LILACS foi realizada por meio do refinamento com os itens: coping, adaptação psicológica, esgotamento profissional, enfermagem e os demais critérios de inclusão supracitados.

Para a busca de artigos na SciELO utilizamos os descritores coping, estresse ocupacional e enfermeiros e a filtragem com o assunto: human sciences.

Foram identificados na LILACS para o descritor coping, estresse ocupacional e enfermeiros respectivamente: 1127, 770 e 3580 artigos. Na SciELO para os descritores coping, estresse ocupacional e enfermeiros foram identificados sequencialmente: 776, 56 e 1552 artigos.

Na LILACS, para os descritores coping, estresse ocupacional e enfermeiros realizando o refinamento, foram identificados, respectivamente, 144, 198 e 139 artigos. Na Embase, para os descritores estresse e enfermeiros foram identificados 7 artigos. Na SciELO, para os descritores coping, estresse ocupacional e enfermeiros foram identificados na devida ordem: 262, 63 e 17, todos filtrados utilizando o assunto, human sciences.

Considerando os critérios de inclusão e exclusão, foram selecionados 12 artigos na LILACS, sendo para o descritor coping 3, estresse ocupacional 8 e enfermeiros um artigo. Na Embase foram selecionados 3 artigos. Na SciELO foi selecionado um artigo para coping, não foram selecionados artigos para os descritores estresse ocupacional e enfermeiros, totalizando, 16 artigos.

Os resultados estão apresentados de forma descritiva, utilizando-se o fluxograma, objetivando a visualização dos dados encontrados. As informações foram extraídas após a leitura dos artigos e, posteriormente, analisadas buscando responder as questões norteadoras.

\section{Resultados}

Considerando os critérios de inclusão e exclusão, foram selecionados 12 artigos na LILACS, 3 artigos na EMBASE e um artigo na SciELO. Os artigos selecionados foram obtidos na íntegra e estavam de acordo com o problema da pesquisa e objetivo do estudo. Os artigos identificados nos dois bancos de dados foram contabilizados somente uma vez, no banco de dados com o maior número de artigos selecionados (figura 1 ).

\section{Discussão}

A amostra é composta por 16 artigos, que abordam os fatores estressantes e as estratégias de coping utilizadas por enfermeiros de ambientes hospitalares no enfrentamento do estresse laboral. 


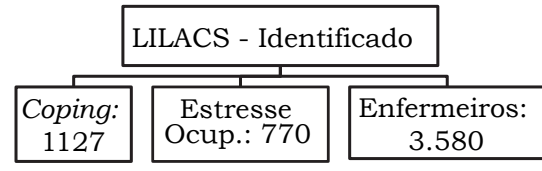

Quantitativo dos artigos após o refinamento
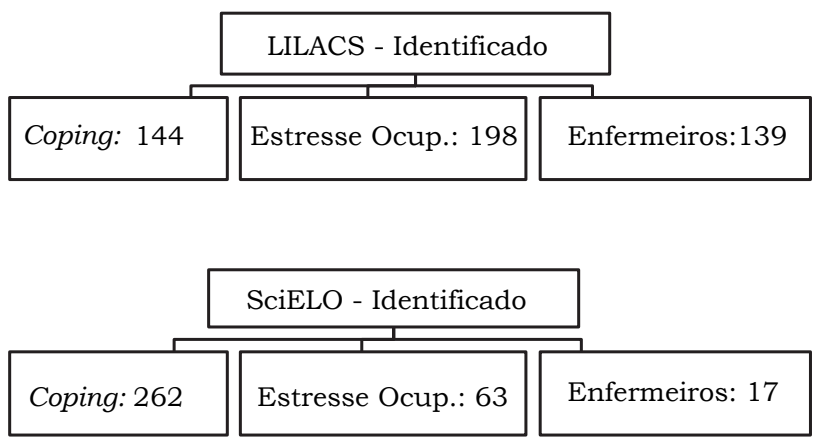

Seleção dos artigos após os critérios de inclusão e exclusão
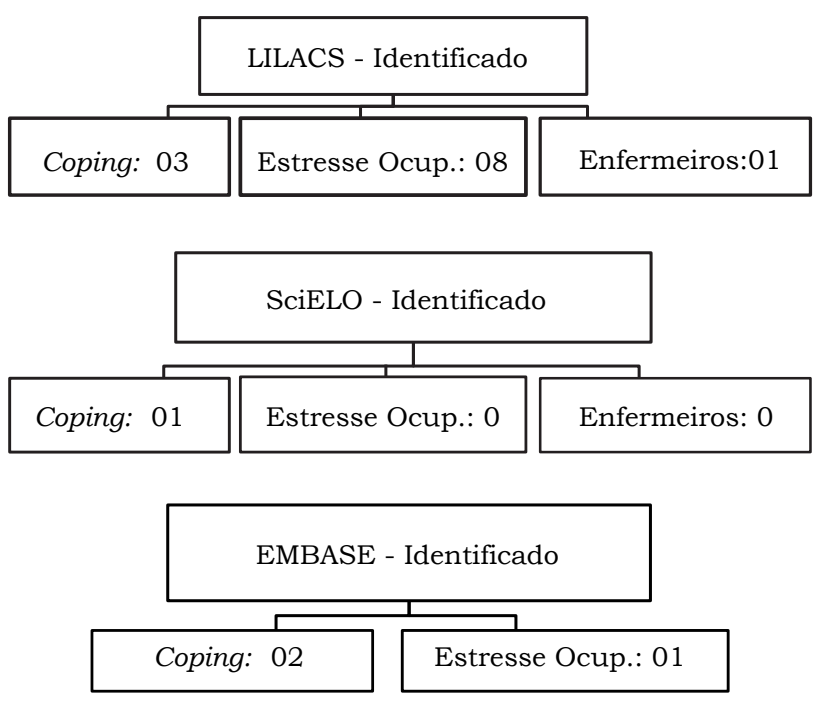

\section{Total de artigos selecionados: 16}

Figura 1. Resultados encontrados na seleção dos artigos nos bancos de dados LILACS e SciELO

FOnTE: pesquisa em bancos de dados online, 2013 
A presença do profissional enfermeiro no ambiente hospitalar é necessária durante a maior parte do tempo, sendo este profissional um dos mais vulneráveis ao estresse. O estresse ocupacional é resultante de mudanças experimentadas pelos trabalhadores devido ao progresso tecnológico e a modificação constante das exigências do mercado de trabalho, e quando essas não se ajustam às necessidades ou capacidades do trabalhador. O organismo, frente a uma situação estressora, procura adaptar-se para que não sofra consequências físicas, psíquicas e biológicas, nascendo, nesse ponto, as estratégias de enfrentamento ao estresse, o coping (5-7).

Partindo de uma análise mais ampla, o estresse é um processo que envolve vários fatores, que podem estar direta ou indiretamente ligados ao processo de trabalho, sendo gerados por uma alta demanda do público e a baixa disponibilidade de materiais e recursos humanos, promovendo um fator de risco para o estresse ocupacional. Dentro do processo de trabalho de enfermagem, são fatores agravantes: o trabalho monótono, em alguns setores; a exigência de atenção constante; o desdobramento das duplas ou triplas jornadas de trabalho; pelo reduzido número de trabalhadores e excesso de tarefas; dois ou mais vínculos empregatícios; pelos baixos salários, realização de horas extras, dentre outros fatores que estão presentes no cotidiano do enfermeiro hospitalar, afetando diretamente sua qualidade de vida $(5,8)$.

O cotidiano hospitalar expõe o enfermeiro a situações geradoras de estresse que estão relacionadas à assistência direta ao paciente como o convívio com a dor e a morte, que podem levar a fadiga e tensão. Além dessas, estão associadas ao estresse, as formas de organização do trabalho, falta de autonomia, supervisão controladora, prolongadas jornadas de trabalho, rotatividade dos setores e o baixo reconhecimento da profissão $(6,8-9)$.

Em unidades fechadas, como a hemodinâmica, identificaram-se como principais circunstâncias favoráveis ao surgimento do estresse: a sobrecarga de trabalho, profissionais com baixa competência e intermediação de conflitos. Os enfermeiros que apresentaram elevados indices de estresse foram os que fazem esforço para ir ao trabalho e que estão insatisfeitos com a profissão. Já os que possuem maior experiência profissional, que se sentem satisfeitos com a sua profissão e que administram melhor os conflitos obtiveram indices baixos de estresse (10).

Em se tratando de unidade de emergência clínica, o estresse está relacionado à carga de trabalho semanal. Aqueles que trabalham somente na emergência apresentaram menor índice de estresse em relação àqueles que trabalham acima de 40 horas semanais em setores diferentes. Além da carga horária foram apontados como fatores estressantes a dificuldade relacionadas com clientes e o processo organizacional (11).

Os enfermeiros de UTI pediátrica possuem um maior comprometimento com o trabalho em relação aos enfermeiros da UTI neonatal, em que as exigências são altas, mas as recompensas também o são, podendo levar a comportamentos de irritabilidade e competitividade entre a equipe de trabalho. Esse resultado reforçou outro estudo no qual diz que o excesso de envolvimento com o trabalho é considerado um fator que pode contribuir com 
o desequilíbrio esforço-recompensa, potencializando seus efeitos nocivos à saúde e ao bem-estar $(12,13)$.

Diferentemente dos estudos supracitados, uma pesquisa realizada com enfermeiros dos hospitais do Brasil mostrou que aqueles que optaram pela unidade em que trabalhavam, não tinham outro vínculo empregatício e que estavam no mesmo setor há um longo período apresentaram baixo nivel de estresse, por que quanto maior o tempo de trabalho no mesmo setor menor o estresse, pois o profissional desenvolve segurança técnica e controle sobre as situações estressoras (14).

$\mathrm{Na}$ tentativa de enfrentar o estresse existem técnicas denominadas coping, que são definidas como esforços cognitivos e comportamentais que visam reduzir a situação avaliada como estressora ao indivíduo. Os tipos de coping citados nos estudos são: suporte social, que é uma forma de enfrentamento, em que o indivíduo busca apoio no coletivo para enfrentar as situações estressoras. Resolução de problemas visa o planejamento de ações que buscam resolver os problemas. Autocontrole baseia-se na manutenção do equilíbrio, afim de não agir por impulso. Afastamento e fuga-esquiva, que é a procura pelo distanciamento da situação, até mesmo a sua não resolução. Confronto é entendido como forma de enfrentamento no qual o indivíduo tenta combater com esforços contrários. Reavaliação positiva é a forma de enfrentamento onde o indivíduo tenta reorganizar a situação, visando amenizar a carga emotiva dos problemas. Aceitação de responsabilidade é a estratégia na qual o profissional assume sua culpa em determinada situação na tentativa da sua resolução $(15,16)$.

$\mathrm{Na}$ área de saúde mental, os métodos usados com maior frequência para encarar o estresse desenvolvido no cuidado aos usuários em sofrimento mental foram o suporte social, seguido da resolução de problemas e autocontrole, esse resultado mostra que os profissionais buscaram maior apoio e ajuda no relacionamento com o outro, seguido de esforços para planejar ações e para resolver problemas e na regulação dos sentimentos e ações frente a situações críticas que caracteriza os fatores citados acima consecutivamente (15).

Em oposição ao estudo citado anteriormente, as estratégias utilizadas entre os enfermeiros dos hospitais do Brasil foram: resolução de problemas, o que leva a pensar que o enfrentamento desses enfermeiros ocorre por meio resolutivo, onde eles definem o problema e planejam a ação, resultando em estratégias efetivas que repercutem em baixo nível de estresse. O menos utilizado foi o fator confronto. Inferindo-se, assim, que não existe coping efetivo ou não, pois a escolha de diferentes estratégias dependerá do indivíduo (14).

Os métodos de enfrentamento frequentemente utilizados por enfermeiros nos cuidados clínicos da dor em ordem crescente são: fuga-esquiva, confronto, autocontrole, resolução de problemas, afastamento, suporte social, aceitação de responsabilidade e reavaliação positiva. Permitindo assim inferir que as estratégias de enfrentamento podem se apresentar de diferentes formas, dependendo da situação em que o profissional é submetido (17).

Em setores como a oncologia, as técnicas mais utilizadas foram as focadas na emoção, essencialmente a reavaliação positiva, onde o indivíduo 
tenta reestruturar o acontecimento na tentativa de amenizar a carga emotiva dos problemas. Outro fator muito utilizado foi à resolução de problema e o utilizado com menor frequência foi a aceitação da responsabilidade (16).

Enfermeiros educadores precisam considerar como as experiências não contribuem apenas para a existência de sofrimento, mas para o eustress. Como educadores interagem com seus alunos e como eles dão feedback $e$ oferece oportunidades importantes para promover a auto eficácia e fornecer um apoio valioso (18). Iniciativas para promover o apoio e auto-eficácia tendem a oferecer benefícios imediatos para o bem-estar dos alunos (19).

A estratégia mais comum usada pelos estudantes foi o comportamento de resolução de problemas, seguido por ficar otimista, prestar atenção e transferência da situação estressante para outras coisas enquanto a evasão foi a menos frequente a ser utilizada (20).

A estratégia Manejo de Sintomas tem impacto positivo sobre o estresse dos enfermeiros de hemato-oncologia uma vez que é efetiva para minimizálo neste setor de trabalho hospitalar, onde a reduzida perspectiva de cura e a cronicidade dos pacientes são inerentes ao trabalho e dificultam ações proativas e de evitação (21).

A maioria dos enfermeiros do estudo apresentou nível médio e alerta para estresse, observando-se que os maiores índices de estresse estão relacionados à administração de pessoal e nas condições de trabalho para o desempenho do enfermeiro (22), mas os causadores máximo de estresse entre os enfermeiros são a baixa remuneração e a falta de estabilidade no emprego (23). O ambiente físico da unidade, nível de barulho, realização de atividades burocráticas e tarefas em tempo minimo apareceram como fatores estressantes (24).

Entre as estratégias de enfrentamento ao estresse utilizadas pela equipe de enfermagem do pronto atendimento são: evitamento, mecanismo que levam a tentar esquecer os estressores; o confronto direto que consiste em falar sobre o assunto e negociar alternativas e o enfrentamento indireto que utiliza de atividades religiosas ou esportivas para aliviar o grau de tensão do estresse (25).

No norte de Portugal, estudos mostraram que os principais fatores favoráveis ao estresse estão relacionados com as implicações de erros cometidos, relação com os profissionais, com os clientes, a carreira profissional, excesso de trabalho e gestão de tempo (26). A insatisfação da equipe de enfermagem na UTI foi atribuída a não poder colaborar mais para a recuperação do paciente, revelando assim, a impotência diante dos limites do tratamento. Para a equipe os recursos afetivos e materiais são amenizadores do estresse no trabalho (27). O gerenciamento de pessoal foi outro fator listado, pois número reduzido de funcionários, cumprimento de tarefas burocráticas de enfermeiros apresentaram-se como fatores de alto nivel de estresse. E a sobrecarga de trabalho quando mantida continuamente determina estresse no enfermeiro atuante em UTI neonatal causando insatisfação pessoal e profissional (28).

O estudo mostrou que os enfermeiros percebem a UTI como fonte mediana de estresse o que indica que não corresponde ao que foi encontrado na 
literatura, pois estudo mostra que a rotina da UTI é um setor de alta tensão o que prejudica a qualidade de vida dos profissionais (29). A sobrecarga de trabalho pode gerar falhas que associados aos conflitos de funções levam a insatisfação nas relações de trabalho e a desvalorização do profissional pode levar a desmotivação ou abandono da atividade laboral (30).

Fatores que influenciam no desenvolvimento do estresse: atividade burocrática, dificuldade em lidar com a terminalidade do paciente oncológico, relação com os familiares, a falta de reconhecimento profissional, sentimento de impotência, a falta de educação permanente e a falta de estratégias que minimizem as consequências fisiológicas e psicológicas causadas pelo estresse (31).

No presente estudo verificou-se uma correlação negativa entre saúde geral e estratégias de coping de evitamento como a auto-distração, negação e descomprometimento, constatou-se a existência de relação entre piores níveis de saúde geral e o recurso a essas estratégias de coping. Isso reforça a ideia de que estratégias centradas no afastamento do indivíduo da fonte de estresse podem ser protetoras em situações pontuais de crise ou de impossibilidade de resolução, mas não são promotoras de equilíbrio físico e emocional em situações que são prolongadas no tempo e que requerem a participação ativa do indivíduo. A aceitação, a auto-distração e a reinterpretação positiva são estratégias de coping focadas na emoção. Esse tipo de coping, quando concentra esforços em si mesmo, para alterar a sua compreensão sobre o estressor e reduzir o mal-estar provocado (32).

A pesquisa Identificou Burnout e alguns fatores associados entre enfermeiros da assistência pediátrica e toco ginecológica de hospital geral do nivel terciário de atenção do Recife (PE) (33).

Quando a estratégia de coping é efetiva, o indivíduo pode solucionar o problema ou diminuir a emoção provocada pela situação, com isso, o estressor poderá ser superado, mas, caso as estratégias sejam ineficazes, haverá continuidade do processo de estresse, o que levará a uma reavaliação do estressor (34). As estratégias utilizadas pelos enfermeiros da unidade no ambiente de trabalho, de acordo com a frequência com que ocorreram são: estabelecer e manter diálogo, colocar-se no lugar do outro, ajuda mútua, de colega, resolver situações conflitantes, buscar aperfeiçoamento profissional, bom humor, calma, atenção, cordialidade, respeito aos funcionários, familiares, pacientes, não transmitir ao paciente o estresse vivenciado e assistir o paciente com qualidade técnica e de forma humanizada, resultando em satisfação pessoal. Com a pesquisa foi possivel inferir que se os enfermeiros estão utilizando estratégias eficazes aos estressores, consequentemente, não há repercussões negativas na assistência ao usuário (35).

No estudo após a análise dos dados, foram construídas sete categorias, de acordo com o agrupamento dos conteúdos referentes ao modo como o estresse ocupacional está presente na vida do enfermeiro que atua no cenário da urgência e emergência, foram elas: escassez de recursos humanos, plantões noturnos, carga horária de trabalho, interface trabalho-lar, relacionamentos interpessoais, recursos materiais e instalações físicas inadequadas, trabalhar em clima de competitividade, distanciamento entre a teoria e a prática (36). 
A estratégia esquiva, relativa às ações e reavaliações que sugerem fuga ou evitação, também contribuiu para a elevação do estresse nos enfermeiros. Por ser focada na emoção, o indivíduo utiliza esse tipo de estratégia com o intuito de alterar a sua compreensão sobre o estressor e reduzir o mal-estar provocado ou mesmo evitar o evento estressor. A utilização sistemática e exclusiva dessa estratégia pode afastar o enfermeiro da realidade que ele precisa confrontar no dia-a-dia, além de não permitir estratégias mais ativas de resolução (32).

O estudo aponta que em unidades abertas e com pacientes adultos os enfermeiros que executam atividades assistenciais estão mais propensos a fatores estressantes. É necessário estar alerta para a necessidade de restaurar o estado de saúde físico e psicológico destes enfermeiros (37).

E mais da metade dos enfermeiros, que assiste pacientes críticos, mostrou sinais de sofrimento físico e/ou psicológico característicos da fase de resistência ao estresse, portanto os achados sugerem a necessidade de atenção a esses profissionais para que seus sintomas não evoluam para a fase de exaustão (38).

No estudo às estratégias de coping identificadas pelos enfermeiros de clínica médica em um hospital universitário, o fator mais utilizado foi à resolução de problemas e a aceitação de responsabilidades o fator menos utilizado (39).

Inúmeros fatores podem desencadear o estresse dentro de um setor de emergência, no estudo são elencados como fatores desencadeadores: condições de trabalho para o desempenho das atividades, o ritmo acelerado de trabalho para a finalização de tarefas associado a poucos recursos humanos. O ambiente associado ao cenário de tensões é visto como fator principal desencadeador do estresse (40).

Analisou-se estresse e estratégias de Coping utilizadas por enfermeiros de Unidade Hemato-Oncológica de um Hospital Universitário do Rio Grande do Sul. O Controle foi o fator mais utilizado para o enfrentamento dos estressores. As estratégias de Coping centradas no problema são consideradas mais efetivas para enfrentar os estressores (41). A administração de pessoal e apontada como maior causadora de estresse. Em relação às estratégias de Coping a Resolução de Problemas é a estratégia mais utilizada pelas enfermeiras da unidade de clínica cirúrgica e a estratégia menos utilizada é Aceitação de Responsabilidades (42).

O estudo sugere que e necessário realização de pesquisas que colaborem com a qualidade de vida desses profissionais, contribuindo na melhora do quadro de estresse (43). As estratégias de enfrentamento elencadas por enfermeiros de unidades críticas foram a participação em eventos que atenuem o efeitos causadores de estresse, como curso educacionais, técnica de relaxamento organizado pela instituição, promovendo uma melhoria na qualidade devida no trabalho (44).

Os estudos apontam que para suavizar e amenizar o estresse, os enfermeiros podem utilizar-se de estratégias de enfrentamento ao estresse, o coping. Dentre essas estratégias citamos o dimensionamento de pessoal 
adequando as demandas do serviço, com implementação de programas de intervenção para trabalhar estresse ocupacional, entre outros (45).

As estratégias de enfrentamento ao estresse gerado pela morte se baseiam principalmente na religião e na espiritualidade, como forma de amenizar e suavizar o estresse gerado por esse acontecimento (46).

O Coping pressupõe a mobilização de recursos por meio dos quais o indivíduo irá empreender esforços cognitivos e comportamentais para gerir as exigências internas ou externas oriundas da interação com o ambiente, avaliadas como ameaça e que excedem as capacidades individuais de confronto (47).

Usualmente, em resposta a um determinado estressor, pode haver utilização conjunta e interdependente desses tipos de Coping. Dessa maneira, o Coping focado na emoção pode facilitar o Coping focado no problema por amenizar a tensão e, similarmente, o Coping focado no problema pode diminuir a ameaça, reduzindo assim a tensão emocional (47).

Quanto às tentativas de resolver os problemas, os médicos tendem a utilizar mais frequentemente o pedido de apoio social, enquanto entre os enfermeiros também surge o sofrimento emocional e a necessidade de evitar o problema (48).

Os resultados sugerem que o aumento da sensação de controle e competência emocional ajudar estudantes de enfermagem a adotar estratégias de enfrentamento ativas e eficazes quando se lida com o estresse, que por sua vez aumenta o seu bem- estar subjetivo. Este estudo destaca o valor potencial de facilitar a inteligência emocional de estudantes de enfermagem e outros profissionais de saúde (49).

Um programa de enfrentamento do estresse baseada em meditação mindfulness foi uma intervenção eficaz para estudantes de enfermagem para diminuir o stress e ansiedade, e pode ser usado para gerenciar o estresse em estudantes de enfermagem (50).

Destaca-se que uma estratégia de Coping não deve ser considerada como intrinsecamente adaptativa ou mal adaptativa, pois é necessário considerar a natureza do estressor, a disponibilidade de recursos de Coping e o resultado do esforço de Coping (21).

\section{Considerações finais}

De acordo com as análises dos artigos pesquisados, percebe-se que independente do setor hospitalar, os principais fatores estressantes são alta demanda do trabalho, baixo reconhecimento profissional, jornada dupla de trabalho, mais de um vínculo empregatício e baixos salários. Quanto às estratégias de coping, a mais utilizada, é a estratégia de resolução de problemas.

Os estudos inferem que o estresse é entendido como algo natural, inerente à condição humana e, para que não se torne patológico, existem as estratégias de coping, compreendidas como medidas que tentam suavizar e amenizar situações estressoras, que dependem das características individuais e organizacionais, mas quando são utilizadas isoladamente, não tornam um coping efetivo, pelo contrário, permitem, assim, a manutenção do estresse. 
Evidenciou-se por meio das análises dos artigos, que as pesquisas que abordam os fatores estressantes entre os enfermeiros são vastas, no entanto, ainda existem lacunas de pesquisas que avaliam as estratégias de coping mais utilizadas por esses profissionais, que visem colaborar com medidas preventivas do estresse.

\section{Conflicto d e interés}

Ninguno

\section{Financiación}

Ninguno

\section{Referências}

1. Schmidt DRC, Dantas RAS, Marziale MHP, Laus AM. Estresse ocupacional entre profissionais de enfermagem do bloco cirúrgico. Texto Contexto-Enferm [internet]. 2009;18(2):330-7.

2. Hanzelmann RS, Passos JP. Imagens e representações da enfermagem acerca do stress e sua influência na atividade laboral. Rev Esc Enferm USP. 2010;44(3):694-701.

3. Moreno FN, Gil GP, Haddad MCL, Vannuchi MTO. Estratégias e intervenções no enfrentamento da Síndrome de Burnout. Rev Enferm UERJ, Rio de Janeiro. 2011;19(1):140-5.

4. Mendes KDS, Silveira RCCP, Galvão CM. Revisão integrativa: método de pesquisa para a incorporação de evidências na saúde e na enfermagem. Texto Contexto-Enferm. 2008 Out-Dez;17(4):758-64.

5. Panunto MR, Guirardello, EB. Carga de trabalho de enfermagem em uma unidade de gastroenterologia. Rev Latino-Am Enfermagem [internet]. 2009 [cited 2013 jul 11];17(6):1009-14. Available from: http:// www.scielo.br/pdf/rlae/v17n6/pt_13.pdf.

6. Mininel VA, Baptista PCP, Felli VEA. Cargas psíquicas e processos de desgaste em trabalhadores de enfermagem de hospitais universitários brasileiros. Rev Latino-Am Enfermagem [internet]. 2011 [cited 2013 jul 11];19(2):340-7. Available from: http://www.scielo.br/pdf/ rlae/v19n2/pt_16.pdf.

7. Cavalheiro AM, Moura Junior DF, Lopes AC. Estresse de enfermeiros com atuação em unidade de terapia intensiva. Rev Latino-Am Enfermagem [internet]. 2008 [cited 2013 jul 11];16(1):29-35. Available from: http://www.scielo.br/pdf/rlae/v16n1/pt_04.pdf.

8. Secco IAO, Robazzi MLCC, Souza FEA, Shimizu DS. Cargas psíquicas de trabalho e desgaste dos trabalhadores de enfermagem de hospital de ensino do Paraná, Brasil. SMAD. Rev Eletrônica Saúde Mental Álcool Drogas [Internet]. 2010 [cited 2013 jul 11]; 6(1):1-17. Available from: http://pepsic.bvsalud.org/pdf/smad/v6n1/16.pdf.

9. Lorenz RV, Benatti MCC, Sabino MO. Burnout e estresse em enfermeiros de um hospital universitário de alta complexidade. Rev Latino-Am Enfermagem [internet]. 2010 [cited 2013 jul 11];18(6):1084-91. Available from: http://www.scielo.br/pdf/rlae/v18n6/pt_07.pdf. 
10. Linch GFC, Guido LA. Estresse de enfermeiros em unidade de hemodinâmica no Rio Grande do Sul, Brasil. Rev Gaúcha Enferm, Porto Alegre (RS). 2011;32(1):63-7.

11. Panizzon C, Luz AMH, Fensterseifer LM. Estresse da equipe de enfermagem de emergência clínica. Rev Gaúcha Enferm, Porto Alegre (RS). 2008 set;29(3):391-9.

12. Fogaça MC, Carvalho WB, Cítero VA, Nogueira-Martins LA. Estudo preliminar sobre o estresse ocupacional de médicos e enfermeiros em UTI pediátrica e neonatal: o equilíbrio entre esforço e recompensa. Rev Latino-Am Enfermagem [Internet]. 2010 [cited 2013 jul 12];18(1):6772. Available from: http://www.scielo.br/pdf/rlae/v18n1/pt_11.pdf.

13. Griep RH, Rotenberg L, Landsbergis P, Silva PRV. Uso combinado de modelos deestresse no trabalho e a saúde auto-referida na enfermagem. Rev Saude Publica 2011;45(1):145-52.

14. Guido LA, Linch GFC, Pitthan LO, Umann J. Estresse, coping e estado de saúde entre enfermeiros hospitalares. Rev Esc Enferm USP. 2011;45(6):1434-9.

15. Santos AFO, Cardoso CL. Profissionais de saúde mental: estresse, enfrentamento e qualidade de vida. Psicologia: Teoria e Pesquisa. 2010;26(3):543-8.

16. Rodrigues AB, Chaves EC. Fatores estressantes e estratégias de coping dos enfermeiros atuantes em oncologia. Rev Latino-Am Enfermagem [internet]. 2008 [cited 2013 jul 12];16(1):24-8. Available from: http:// www.scielo.br/pdf/rlae/v16n1/pt_03.pdf.

17. Negromonte MRO, Araujo TCCF. Impacto do manejo clínico da dor: avaliação de estresse e enfrentamento entre profissionais de Saúde. Rev Latino-Am Enfermagem [Internet]. 2011 [cited 2013 jul 12];19(2):23844. Available from: http://www.scielo.br/pdf/rlae/v19n2/pt_03.pdf.

18. Gibbons C. Stress, coping and burn-out in nursing students. Int J Nurs Stud. 2010;47:1299-1309.

19. Gibbons C, Dempster M, Moutray M. Stress, coping and satisfaction in nursing students. J Adv Nurs. 2010;67:621-32.

20. Shaban I, Khater W, Akhu-Zaheya L. Undergraduate nursing students' stress sources and coping behaviors during their initial period of clinical training: a Jordanian perspective. Nurse Educ Pract. 2012;12:204-9.

21. Umann J, Silva RM, Benavente SBT, Guido LA. O impacto das estratégias de enfrentamento na intensidade de estresse de enfermeiras de hemato-oncologia. Rev Gaúcha Enferm. 2014;35(3):103-10.

22. Guerrer FJL, Bianchi ERF. Caracterização do estresse nos enfermeiros de unidades de terapia intensiva. Rev Esc Enferm USP. 2008;42(2):355-62.

23. Mizobuchi LEC, Cury CFMR. Estresse na enfermagem: mensuração das situações geradoras em um hospital geral. Rev Inst Ciênc Saúde. 2007;25(4):349-55.

24. Lima GF, Simonetti SH, Bianchi ERF, Kobayashi RM. Caracterização do estresse de enfermeiros que atuam em hospital especializado em cardiologia. Enfermería Global. 2012;N28. 
25. Calderero ARL, Miasso AI, Corradi-Webster CM. Estresse e estratégias de enfrentamento em uma equipe de enfermagem de pronto atendimento. Revista Eletrônica de Enfermagem [internet]. 2008;10(1):51-62. Available from: http://www.fen.ufg.br/revista/v10/n1/v10n1a05.htm.

26. Gomes AR, Cruz JF, Cabanelas S. Estresse ocupacional em profissionais de saúde: um estudo com enfermeiros portugueses. Psicologia: Teoria e Pesquisa. 2009;25(3):307-18.

27. Lucas JS, Passos JP. O estresse no trabalho da equipe de enfermagem em unidade de terapia intensiva. Rev de Pesq: Cuidado é Fundamental. 2009;1(2):345-52.

28. Anjos DR, Silva EA, Falqueiro HJA, Freitas PMP, Peres VPM, Massruhá VC, et al. Estresse: fatores desencadeantes, identificação e avaliação de sinais e sintomas no enfermeiro atuante em UTI neonatal. Rev Inst Ciênc Saúde. 2008;26(4):426-31.

29. Murassaki ACY, Versa GLGS, Inoue KC, Melo WA, Matsuda LM. Estresse em enfermeiros intensivistas e a condição chefe/não chefe de família. Cienc Cuid Saude. 2011;10(4):755-62.

30. Santos FD, Cunha MHF, Robazzi MLCC, Pedrão LJ, Silva LA, Terra FS. O estresse do enfermeiro nas unidades de terapia intensiva adulto: uma revisão da literatura. SMAD. Revista Eletrônica Saúde Mental Álcool e Drogas. 2010;6(1):1-16.

31. Hercos TM, Vieira FS, Oliveira MS, Buetto LS, Shimura CMN, Sonobe HM. O trabalho dos profissionais de enfermagem em unidades de terapia intensiva na assistência ao paciente oncológico. Rev Bras Cancerol. 2014;60(1):51-8.

32. Gomes SFS, Santos MMMCC, Carolino ETMA. Riscos psicossociais no trabalho: estresse e estratégias de coping em enfermeiros em oncologia. Rev Latino-Am Enfermagem [internet]. 2013 [citado $2013 \mathrm{dez}$ 13];21(6):1282-9. Available from: http://www.revistas.usp.br/rlae/ article/view/76051/79740.

33. Galindo RH, Feliciano KVO, Lima RAS, Souza AI. Síndrome de burnout entre enfermeiros de um hospital geral da cidade do Recife. Rev Esc Enferm USP. 2012;46(2):420-7.

34. Kleinubing RE, Goulart CT, Silva RM, Umann J, Guido LA. Estresse e coping em enfermeiros de terapia intensiva adulto e cardiológica. Rev Enferm UFSM [internet]. 2013;3(2):335-44. Available from: http://cascavel.ufsm.br/revistas/ojs-2.2.2/index.php/reufsm/article/ view/8924/pdf.

35. Silveira MM, Stumm EMF, Kirchner RM. Estressores e coping: enfermeiros de uma unidade de emergência hospitalar. Rev Eletr Enf [Internet]. 2009;11(4):894-903.

36. Bezerra FN, Silva TM, Ramos VP. Estresse ocupacional dos enfermeiros de urgência e emergência: Revisão Integrativa da Literatura. Acta Paul Enferm. 2012;25(especial 2):151-6.

37. NegeliskiI C, Lautert L. Estresse laboral e capacidade para o trabalho de enfermeiros de um grupo hospitalar. Rev Latino-Am Enfermagem. 2011;19(3):[08 telas]. 
38. Ferrareze MVG, Ferreira V, Carvalho AMP. Percepção do estresse entre enfermeiros que atuam em Terapia Intensiva. Acta Paul Enferm. 2006;19(3):310-15.

39. Guido LA, Umann J, Stekel LMC, Linch GFC, Silva RM, Lopes LFD. Estresse, Coping e estado de saúde de enfermeiros de clínica médica em um hospital universitário. Cienc Cuid Saude. 2009;8(4):615-21.

40. Batista KM, Bianchi ERF. Estresse do Enfermeiro em unidade de emergência. Rev Latino-Am Enfermagem. 2006;14(4):534-9.

41. Umann J, Silva RM, Benetti ERR, et al. Estresse e coping entre enfermeiros de unidade hemato-oncológica. Rev Rene. 2013;14(4):783-90.

42. Guido LA, Silva RM, Goulart CT. Estresse e coping entre enfermeiros de unidade cirúrgica de hospital universitário. Rev Rene [internet]. 2012;13(2):428-36. Available from: http://www.revistarene.ufc.br/revista/index.php/revista/article/view/226/pdf.

43. Rolim CSS. Estresse e sindrome de burnout em profissionais de enfermagem. Rev Bras Pesq Saúde Vitória. 2013;15(3):103-13.

44. Brandão DEC, Galvão CM. O estresse da equipe de enfermagem que atua no período perioperatório: revisão integrativa. Rev Rene. 2013;14(4):836-44.

45. Decezaro A, Frizon G, Silva OM, Toniolo CL, Busnelo, GF, Ascari RA. $O$ estresse dos enfermeiros que atuam na unidade de terapia intensiva: uma revisão de literatura. Revista UNINGÁ. 2014;19(2):29-32.

46. Rockembach J, Casarin ST, Siqueira HC. Morte pediátrica no cotidiano de trabalho do enfermeiro: sentimentos e estratégias de enfrentamento. Rev Rene. Fortaleza. 2010;11(2):63-71.

47. Lazarus RS, Folkman S. Stress, appraisal and coping. New York: Springer Publishing Company; 1984.

48. Giansante M, Ballarini V. Perceived stress and coping strategies in medical and nursing staff in a department of clinical hematology: pilot study. G Ital Med Lav Ergon. 2012;34(2 Suppl B):B23-8.

49. Por J, Barriball L, Fitzpatrick J, Roberts J. Emotional intelligence: Its relationship to stress, coping, well-being and professional performance in nursing students. Nurse Educ Today. 2011;31(8):855-60.

50. Kang YS, Choi SY, Ryu E. The effectiveness of a stress coping program based on mindfulness meditation on the stress, anxiety, and depression experienced by nursing students in Korea. Nurse Educ Today. 2009;29(5):538-43. 
